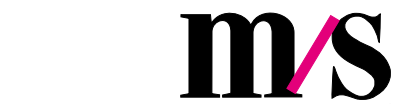

médecine/sciences $1999 ; 15: 447-50$
Richard S.J. Frackowiak*

\title{
NEURO-IMAGERIE FONCTIONNELLE : UNE RÉVOLUTION EN NEUROSCIENCES
}

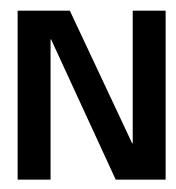

ous vivons une époque dans laquelle la plupart des gens sont convaincus que notre vie émotionnelle, intellectuelle et même biologique est entièrement déterminée par notre cerveau. Néanmoins, comme s'il s'agissait d'une séquelle de l'époque à laquelle on pensait que le siège de l'esprit se trouvait au niveau du cœur ou de l'épiphyse, nous continuons de discourir de nos émotions, intentions et actions en des termes qui esquivent délibérément toute référence à la biologie du cerveau. L'une des nouvelles quêtes intellectuelles de notre temps est la neurophilosophie. Elle vise à réconcilier les faits biologiques fondamentaux du cerveau avec des concepts abstraits qui décrivent le comportement humain en termes de références religieuses ou philosophiques. Cette situation relève de deux facteurs distincts. Le premier consiste en une limite imposée par notre manque d'imagination dans la formulation des attributs du comportement humain en termes biologiques, et notamment de physiologie et de chimie du cerveau. Le second facteur a été notre remarquable incapacité technique à décrire de façon détaillée l'anatomie fonctionnelle du cerveau humain. Cette hypothèque est aujourd'hui levée grâce à l'introduction de méthodes puissantes d'enregistrement atraumatique du fonctionnement cérébral. Je crois fermement que l'acquisition de nouvelles données concernant la façon dont notre cerveau est organisé pour produire les multiples facettes du comportement humain nous donnera bientôt une nouvelle vision de notre vie consciente, et nous entraînera loin de ces discussions sans fin et sans objet à propos de la capacité même du cerveau à s'analyser luimême. Il s'agit là d'une entreprise d'une importance fondamentale dans la société moderne, et qui ne saurait être niée par personne. Avec le vieillissement de nos populations et l'expansion de maladies neurodégénératives conduisant à une destruction progressive de la personnalité et de l'humanité des individus, nous devons comprendre mieux le cerveau de façon à avoir un espoir de lutter contre ces processus de détérioration. Il en existe néanmoins qui sont effrayés par une telle perspective. En effet, la possibilité qu'un jour, grâce à une meilleure compréhension des mécanismes cérébraux, nous puissions être manipulés par des forces malveillantes, inquiète les chercheurs autant que les non-scientifiques. Pourtant, la manipulation de la conscience sociale et des aspirations individuelles est déjà un fait de l'existence. L'impact des images à travers le cinéma et la télévision, du son à travers la stéréophonie et la radio, et la propagande au travers des médias populaires et de la littérature, sont autant d'exemples de modification de la pensée et des sentiments humains ayant un grand impact individuellement sur chacun de nous à travers des mécanismes cérébraux communs. Comment une compréhension plus fine de nos cerveaux et de la façon dont ils sont organisés entraînerait des influences encore plus malveillantes sur notre comportement n'est pas absolument évident. En effet, beaucoup opposeraient même l'idée que l'inverse serait plus 
vraisemblable. Ce genre d'argument ne pourra cependant être valablement avancé que lorsque notre connaissance sera plus assurée, plutôt que dans le climat d'ignorance actuel.

Pourquoi savons-nous si peu de la structure fonctionnelle du cerveau humain, par comparaison à d'autres organes comme par exemple le rein, le foie ou le coeur? Cette question mérite d'être posée, et pourtant la réponse est presque banale. D'abord, comme ces autres organes, le cerveau est en apparence homogène, du moins à l'œil nu, mais il est beaucoup moins accessible chez le sujet vivant. En effet, le cerveau, qui pèse approximativement 1400 grammes en moyenne, est contenu à l'intérieur du crâne, une boîte osseuse essentiellement impénétrable. De façon encore plus problématique, l'idée même de prélever du tissu cérébral pour l'examiner au microscope ou faire d'autres études anatomiques ou physiologiques, peut entraîner des perturbations importantes du comportement qui rendent ces biopsies éthiquement inacceptables chez le sujet vivant. De ce fait, la science du fonctionnement cérébral dépendait essentiellement d'inférences faites à partir d'observations de comportements anormaux en corrélation avec la topographie et la taille des lésions cérébrales déterminées post-mortem, puis, plus récemment, par imagerie anatomique in vivo (voir ci-dessous). Bien que cette méthode ait été extrêmement fructueuse pour engendrer des hypothèses sur l'organisation fonctionnelle du cerveau au cours des 120 dernières années, il y a néanmoins un problème de logique avec cette approche. En effet, son interprétation dépend de l'hypothèse selon laquelle le cerveau humain se compose de modules indépendants et fonctionnellement distincts, qui peuvent être retirés séparément sans perturber le fonctionnement des parties laissées intactes. Or, on sait à présent que ce n'est pas le cas. Le cerveau lésé, comme le cerveau normal en développement, peut s'adapter et se réorganiser fonctionnellement. Par exemple, l'expérience clinique

hémiplégie d'origine vasculaire récupèrent souvent de façon spontanée des fonctions neurologiques sur de longs mois après l'accident vasculaire initial. De même, les régions du cortex cérébral qui reçoivent les signaux sensitifs d'un hémicorps peuvent traiter les signaux venant d'autres parties du corps dans certaines circonstances.

L'étude régionale du fonctionnement cérébral chez l'homme peut être effectuée au moyen de diverses techniques, chacune d'entre elles fournissant une information unique. Chaque technique a ses forces et ses faiblesses, et beaucoup d'entre elles sont encore dans une phase de développement. Dans certains cas, il y a encore besoin de progresser au niveau de l'instrumentation ou bien de l'analyse des données afin de transformer une technique potentiellement utile en un instrument fiable sur le plan scientifique. Néanmoins, c'est seulement depuis le développement de techniques atraumatiques permettant d'enregistrer le fonctionnement du cerveau humain vivant qu'une révolution s'est opérée dans notre capacité à produire de grandes quantités de données objectives concernant son organisation fonctionnelle. Cela a ouvert un chapitre dans l'histoire de notre compréhension de nous-mêmes, tel que notre cerveau puisse à présent se pencher sur lui-même au moyen d'une méthode scientifique. Ce chapitre s'ouvre en 1972, avec l'invention du scanner à rayon X (tomodensitométrie) à la suite de la découverte des principes de la tomographie par reconstruction, découverte pour laquelle Hounsfield et McCormack se sont vus décerner le prix Nobel. Dans cette approche, il est possible de reconstruire l'arrangement anatomique du cerveau humain vivant en mettant à profit la propriété des différents tissus cérébraux d'atténuer de façon différentielle les rayons X. La tomodensitométrie engendre des images morphologiques, mais avec un faible contraste entre substance grise et substance blanche du fait de leur faible différence d'atténuation des rayons X. Les images de scanner permettent de différencier nettement le tissu cérébral des tissus envi- ronnants (cerveau, liquide céphalorachidien, sang ou air) et ce de façon beaucoup plus performante que la différenciation substance grise-substance blanche. L'imagerie par résonance magnétique (IRM) permet de produire une gamme d'images morphologiques du cerveau exploitant seulement les propriétés magnétiques des tissus. Ces images sont engendrées grâce à des modifications des paramètres d'enregistrements des données mettant à profit les propriétés différentielles des protons dans la substance grise et la substance blanche du cerveau. La diversité des informations anatomiques obtenues de cette façon, ajouté au fait que ces données sont acquises sans recourir aux radiations ionisantes, font de l'IRM la méthode de choix pour étudier l'anatomie du cerveau humain. Les images peuvent être représentées sous forme de coupes du cerveau selon une orientation anatomique conventionnelle ou si besoin dans n'importe quelle orientation souhaitée. Des images peuvent également être représentées sous forme d'un rendu de surface grâce à un traitement informatique, fournissant une image de la surface du cortex cérébral avec ses circonvolutions et ses sillons. Leur résolution anatomique dans des conditions cliniques habituelles est de l'ordre de $1 \mathrm{~mm}^{3}$. Le principe de la reconstruction tomographique a été appliqué vers la fin des années 1970 pour détecter des quantités infimes de molécules chimiques rendues détectables par des scanners à émission grâce au marquage par des radio-isotopes. Ces molécules, également appelées radiotraceurs, se répartissent dans le cerveau en fonction de leur propriétés chimiques ou physiologiques, au travers de réactions spécifiques. En suivant le devenir de ces molécules dans le tissu cérébral au moyen de tomographes, il devient alors possible d'obtenir une mesure de paramètres régionaux qui reflètent ces réactions. Cette méthode, connue sous le nom de tomographie par émission de positons (TEP), fournit des images extrêmement fiables. De plus, les images du fonctionnement cérébral obtenues par cette méthode peuvent être recalées en trois dimensions avec 
des coupes anatomiques d'IRM, ce qui permet d'étudier de façon précise le fonctionnement du cerveau humain vivant.

Le cerveau est fait de cellules nerveuses qui sont réparties spatialement d'une façon non pas aléatoire mais liée à leurs propriétés fonctionnelles. Ces cellules communiquent les unes avec les autres et transportent des signaux selon des codes et des profils obéissant à une distribution spatiale et à un déroulement temporel bien déterminés. Les fonctions du cerveau peuvent donc être décrites selon plusieurs niveaux d'agrandissement, que ce soit dans le domaine temporel ou spatial. Au niveau spatial, les neurones, les regroupements neuronaux, et les populations neuronales travaillant à un niveau macroscopique de façon fonctionnellement homogène ou coordonnée, représentent différents niveaux de l'organisation cérébrale qui sont accessibles par les techniques décrites ci-dessus. Parallèlement, des profils d'activité électrique cérébrale avec des périodes temporelles de l'ordre de la milliseconde, de la centaine de millisecondes, voire de plusieurs secondes, ont été décrits. Par exemple, le potentiel d'action, qui est l'unité de base de la transmission du signal électrique dans le cerveau, a une durée de quelques millisecondes; le potentiel évoqué, qui exprime une activité électrique intégrée d'origine néo-corticale en réponse à un stimulus expérimental, se mesure en centaines de millisecondes ; le potentiel de préparation (variation contingente négative) et l'onde $\delta$ de l'électroencéphalogramme sont des signaux spontanés ou évoqués qui durent plusieurs secondes. Des méthodes telles que l'électroencéphalographie ou la magnétoencéphalographie qui enregistrent respectivement les champs de potentiels électriques ou les potentiels magnétiques qui accompagnent ceux-ci, permettent d'obtenir une résolution temporelle de l'ordre de la milliseconde. Ces techniques, combinées aux techniques d'imagerie morphologique et fonctionnelle décrites ci-dessus ont permis de mettre ces différents niveaux de l'organisation cérébrale à notre por- tée, et fournissent un moyen d'analyser de façon systématique l'architecture fonctionnelle du cerveau humain à la fois sur un plan spatial et sur un plan temporel.

L'objectif des neurosciences de l'image est de montrer comment fonctionne le cerveau. Le cerveau est en apparence uniforme sur toute sa surface (le cortex), mais il existe des différences considérables dans l'orientation cellulaire et l'arrangement des couches cellulaires dans les différentes parties du cortex. La spécialisation fonctionnelle que traduisent ces différences morphologiques est encore très mal connue. Les régions cérébrales sont activées de façon différente pendant des tâches distinctes. L'organisation fonctionnelle du cerveau peut donc être décrite en termes de disposition de regroupements neuronaux qui eux-mêmes peuvent être organisés en réseaux interfacés et en systèmes fonctionnels intégrés. Ce dernier niveau, intégré, a pour objectif de décrire comment les fonctions cérébrales sont inscrites dans la structure physique du cerveau. Dans ce numéro de médecine/ sciences, différents exemples seront présentés pour illustrer le type de question qui peut être étudiée et quelles problématiques peuvent être abordées. Comment les données sensorielles primaires telles que le toucher et la vision sont-elles traitées par le cerveau humain? A quel moment les cartes de signaux nerveux sontelles engendrées ? Comment les perceptions sont-elles reconnues, engendrées, nommées et utilisées pour guider le comportement? Comment les profils temporels d'activité neuronale qui représentent des sensations évoquées dans certaines régions cérébrales interagissent-ils avec les régions cérébrales, et comment les profils d'activité neuronale entrâ̂nent-ils en réponse une contraction musculaire produisant le mouvement ? Quels sont les substrats neuronaux de fonctions cognitives telles que la mémoire, le langage et l'émotion? Ces fonctions sont-elles le résultat d'activités neuronales dans des régions bien précises et hautement spécialisées ou, au contraire dépendent-elles d'interactions entre un grand nombre de régions céré- brales qui ont des fonctions plus fondamentales ou plus modulaires? Dans ce numéro de médecine/sciences seront présentées des données concernant nos fonctions cérébrales qui illustrent la puissance de ces techniques et l'avancée conceptuelle dans le domaine de la psychologie humaine qui résulte de ces progrès. Pour le comprendre, il suffit de dire que les modifications de l'activité cérébrale associées à la pensée pure, l'émotion ou les processus cognitifs sont maintenant tous facilement mesurables. Nous sommes actuellement inondés en grande abondance de données nouvelles concernant l'organisation fonctionnelle de notre organe de la pensée, ce qui, inévitablement, aura un impact considérable sur notre compréhension des processus pathologiques et des stratégies thérapeutiques

\section{RÉFÉRENCES}

1. Baddeley A. Human memory. Erlbaum : Hove, 1990

2. Binder JR, Rao SM, Hammeke TA, et al. Functional magnetic resonance imaging of human auditory cortex. Ann Neurol 1994 ; $35: 662-72$.

3. Boecker H, Kleinschmidt A, Requardt M, Hanicke W, Merboldt KD, Frahm J. Functional co-operativity of human cortical motor areas during self-paced simple finger movements. A high resolution MRI study. Brain 1994 ; 117 : 1231-40.

4. Colebatch JG, Deiber MP, Passingham RE, Friston KJ, Frackowiak RSJ. Regional cerebral blood flow during voluntary arm and hand movements in human subjects. INeurophysiol 1991 ; 65 : 1392-401.

5. Courtney SM, Ungerleider LG, Keil K, Haxby JV. Object and spatial visual working memory activate separate neural systems in human cortex. Cereb Cortex 1996 ; 6 : 39-49.

6. Detre JA, Leigh JS, Williams DS, Koretsky AP. Perfusion imaging. Magn Reson Med $1992 ; 23: 37-45$

7. Frackowiak RSJ. Functional mapping of verbal memory and language. Trends Neurosci $1994 ; 17$ : 109-15.

8. Frackowiak RSJ, Friston KJ, Frith CD, Dolan RJ, Mazziotta JC. Human brain function. San Diego : Academic Press, 1998.

9. Frith CD, Friston KJ, Liddle PF, Frackowiak RSJ. Willed action and the prefrontal cortex in man. Proc R Soc Lond B 1991 ; 244 : 241-6. 


\section{RÉFÉRENCES}

10. Grafton ST, Woods RP, Mazziotta JC, Phelps ME. Somatotopic mapping of the primary motor cortex in humans : activations studies with cerebral blood flow and positron emission tomography. J Neurophysiol 1991 ; $66: 735-43$

11. Grafton ST, Mazziotta JC, Woods RP, Phelps ME. Human functional anatomy of visually guided finger movements. Brain 1992 ; $115: 565-87$.

12. Hari R, Lounasmaa OV. Recording and interpretation of cerebral magnetic fields. Science 1989 ; 244 : 432-6.

13. Hari R. Human cortical functions revealed by magnetoencephalography. Prog Brain Res 1994 ; 100 : 163-8.

14. Hillyard SA. Electrical and magnetic brain recordings : contributions to cognitive neuroscience. Curr Opin Neurobiol 1993 ; 3 : 217-24.

15. Makela JP, Ahonen A, Hamalainen M, et al. Functional differences between auditory cortices of the two hemispheres revealed by whole-head neuromagnetic recordings. Hum Brain Map 1993 ; 1 : 48-56.

16. Posner MI, Raichle ME. Images of the mind. New York : Scientific American Library, 1994.
17. Raichle ME. Circulatory and metabolic correlates of brain function in normal humans. In : Plum F, ed. Handbook of physiology: the nervous system, vol. V. New York : American Physiological Society, Oxford University Press, 1987 : 643-74.

18. Roland PE. Brain activation. New York : Wiley Liss, 1993.

19. Rugg MD. Event related potentials in clinical neuropsychology. In : Crawford JR, Parker DM, McKinley WW, eds. Handbook of neuropsychological assessment. Erlbaum : Hove, 1992.

20. Wilding EL, Doyle MC, Rugg MD. Recognition memory with and without retrieval of context : an event related potential study. Neuropsychologia 1995 ; 33 : 743-67.

21. Seitz RJ, Roland PE. Learning of sequential finger movements in man : a combined kinematic and positron emission tomography (PET) study. Eur J Neurosci 1992 ; 4: 154-65.

22. Stehling MK, Turner R, Mansfield P. Echo-planar imaging : magnetic resonance imaging in a fraction of second. Science 1991 ; 254:43-50.

23. Stephan KM, Fink GR, Passingham RE, et $a l$. Functional anatomy of the mental representation of upper extremity movements in healthy subjects. J Neurophysiol $1995 ; 73$ : 373-86.
24. Tootel RB, Reppas JB, Kwong KK, et al. Functional analysis of human MT and related visual cortical areas using magnetic resonance imaging. J Neurosci $1995 ; 15$ : 3215-30.

25. Tulving E. Elements of episodic memory. Oxford : Oxford University Press, 1983.

26. Zeki SM. A vision of the brain. London : Blackwell Scientific Publication, 1993.

27. Zeki SM, Watson, JD, Frackowiak RSJ. Going beyond the information given : the relation of illusory visual motion to brain activity. Proc R Soc Lond B Biol Sci 1993 ; 252 : 21522

28. Zeki SM, Watson JD, Lueck CJ, Friston KJ, Kennard K, Frackowiak RSJ. A direct demonstration of functional specialization in human visual cortex. J Neurosci 1991 ; 11 : 641-9.

\section{TIRÉS À PART}

R.S.J. Frackowiak. 